\title{
PEGylation and BioPEGylation of Polyhydroxyalkanoates: Synthesis, Characterisation and Applications
}

\author{
John Foster \\ Bio/Polymer Research Group, University of New South Wales \\ Australia
}

\section{Introduction}

Chemical conjugations with poly(ethylene glycols) (PEGs) are established procedures to facilitate solubilisation of hydrophobic compounds. Techniques for PEGylation are currently applied to various hydrophobic pharmaceutical agents for drug delivery. More recently PEGylation has been applied to members of a novel family of microbial biopolyesters: the 'Polyhydroxyalkanoates' (PHAs). Complementing these chemical techniques, PEG modulation of bioprocessing protocols for the production of PHAs has shown that the strategic addition of certain PEGs not only supports hybrid synthesis, but may provide a technique for control of PHA composition and molecular mass. Furthermore, the addition of PEGs to the microbial cultivation systems can also result in end-capping of the hydrophobic polymer chains; a process termed 'bioPEGylation'. In this chapter we review the processes of PEGylation and bioPEGylation of PHAs as well as the influence they exert on the physiochemical, material and biological properties of the resulting natural-synthetic hybrid copolymer materials.

\section{Polyhydroxyalkanoates (PHAs)}

Polyhydroxyalkanoates (PHAs) are a family of microbial polyesters synthesized under conditions of environmental stress with excess of available carbon and one or more limiting nutrients (Doi, 1990; Steinbüchel \& Fuchtenbusch, 1998; Lenz \& Marchessault, 2005;). These biopolyesters are intracellularly sequestered as readily visible white refractile inclusion bodies and are considered to act as carbon reserves and possibly also serve as ion sinks; (Figure 1), (Anderson and Dawes, 1990; Foster, 2000).

First reported by Lemoigne in 1925, Poly(3-hydroxybutyrate), (PHB) is the most studied member of the PHA family and has a track record of commercial success. PHB is a homopolymer comprised of monomer 3-hydroxybutyric acid units (HBA). As one of the ketone bodies, HBA plays the vital role of carbon supply in mammalian systems responding to periods of prolonged starvation or suffering from diabetes mellitus (Bondy et al. 1949). Foster and Tighe have shown that microbial HBA is chemically identical to its mammalian counterpart and is recognised and processed by mammalian enzymes (Foster and Tighe 1995). Martin et al. subsequently patented its use, and that of its oligomers, as therapeutic agents for the treatment of insulin resistant states and appetite control as well as 
neurodegenerative disorders and epilepsy (Martins et al. 1999). In addition, Reusch et al. have reported that mammalian cells also synthesise trace quantities of PHB which is proposed to serve a membrane transport function (Reusch et al. 1992). In fact, such low molecular weight PHB is widely distributed in cells and found in representative organism of nearly all phyla. Complexation of this PHB with a range of other macromolecules modifies the physical and chemical properties and as a consequence, this complexed PHB can also be found in cytoplasm (Reusch et al. 1992).

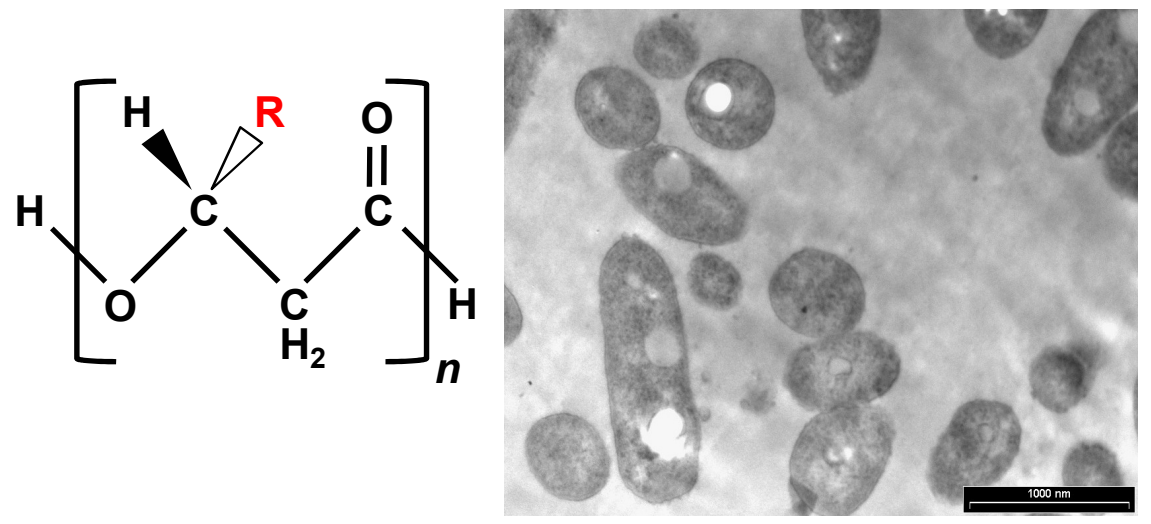

Fig. 1. Chemical formula for poly(3-hydroxyalkanoate)s and transmission electron micrograph PHA refractile inclusion bodies in Pseudomonas oleovorans (bar $=1,000 \mathrm{~nm}$ ).

As a consequence of its biocompatibility, PHB was initially pursued as a commercial biomaterial for various medical devices (Foster, 1996). However, its ability to successfully fulfil such a role is hampered by its relatively high crystallinity and brittle nature which also influence in degradation behaviour in vivo ( $\mathrm{Wu} \& \mathrm{Chen}, 2009)$. These properties can be modified through copolymerisation with hydroxyvalerate to yield P(HB-co-HV) (Lenz, 1993; Yasin \& Tighe 1993). The physiochemical and material properties of $\mathrm{P}(\mathrm{HB}-\mathrm{co}-\mathrm{HV})$ are more commercially appropriate and it is this copolymer which is the focus of much attention as a 'bioplastic'; a biodegradable replacement to conventional thermoplastics (Hänggi, 1995; Steinbüchel, 1996; Lenz \& Marchessault, 2005).

Copolymerisation of PHB with random units of HV occurs through manipulation of bioprocessing systems, usually through variations in carbon feedstock. Further investigations in bioprocessing parameters have shown significant metabolic flexibility in a wide range of microorganisms for the synthesis of a spectrum of PHAs with over 105 different monomeric components. The properties of these PHAs range from the brittle and crystalline, as with PHB, to the flexible and elastomeric found in Poly(3-hydroyoctanoate-coundecylenoate), (PHOU), (Steinbüchel, 1996; Steinbüchel, 2001). PHA synthesis is species dependent and while some species such as Ralstonia eutropha synthesise PHAs with comparatively short chian length substituents in the side chain (scl-PHA), many of the pseudomonads belonging to RNA homology Group I, produce PHAs with medium chain length alkyl groups in the side chain ( $m c l$-PHAs) such as Poly(3-hydroxyoctanoate), (PHO). An additional group may be also be classified as PHAs possessing interesting chemically functional groups in their side chains ( $f$ cl-PHAs), such as cyanophenoxy and phenyl groups (Kim et al. 1995; Foster, 1996; Steinbüchel, 1996; Kim et al. 2000). In addition to variations in 
side chain composition, PHAs with elongation of monomer units in the chain backbone have also been reported, such as Poly(3HB-co-4HB), (Nakamura et al. 1992).

\section{Poly(ethyelene glycol)s (PEGs)}

Poly(ethylene glycol)s (PEGs) are oligomers or polymers of ethylene oxide (Figure 2). These synthetic polymers, produced through acidic or base catalysed polycondensation reactions, constitute a family of neutral, water-soluble polyethers with various molecular weights ranging from the oily, viscous liquid of PEG with a molecular weight of 106, also known as diethylene glycol (DEG, PEG-106), to the waxy, crystalline solid of PEG-4000. PEGs with molecular weights around 25,000 contain no hydroxyl end group and are referred to as Polyethylene oxide (PEO), (Harris, 1992).

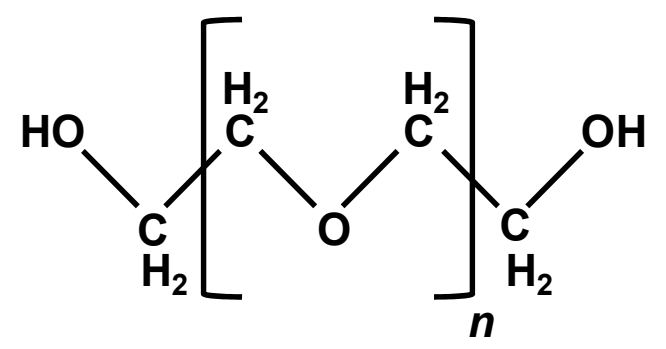

Fig. 2. Chemical formula for poly(ethylene glycol)s.

A variety of PEGs are employed as commodity chemicals in various industrial products ranging from cosmetics and lubricants to plasticisers and antifreeze agents. However some PEGs are biocompatible and utilised in the biomedical field, such as in the preservation of organs and tissues. Because of their hydrophilic nature, PEGs are also applied in the pharmaceutical industry to solubilise hydrophobic components and in protein purification. Protein-PEG conjugates exhibit a longer retention time in blood, this consequently supports their prolonged activity and, in the case of medication, longer dosing intervals. Classic examples include PEG-interferon a for the treatment of hepatitis-C and PEGylated granulocyte colony stimulating factor for neutropenia (Harris, 1992; Harris and Chess, 2003; Steward, 2005). In 1990, Krause and Bittner used PEGs with varying molecular weights to morphologically fuse severed axons in invertebrates and continuing research in this field has demonstrated that PEG can support nerve repair in mammals (Krause and Bittner, 1990; Borgens \& Bohnert, 2001; Laverty et al. 2004).

\section{PEGylation of PHAs}

PEGylation is an established process for attaching or conjugating PEG chains to other molecules, including biomacromolecules such as peptides, proteins and antibody fragments for the production of biopharmaceuticals. PEGylation modifies the physiochemical properties including chain conformation, electrostatic binding and most noticeably, hydrophobicity. Conjugation of PHB with PEGs through chemical routes has been investigated by a number of authors.

Triblock copolymers of PHB-co-PEG-co-PHB have been synthesised by Kumagai et al. and Shuai et al. using ring-opening polymerisation of [R,S]- $\beta$-butyrolactone with PEG based 
macroinitiators. These triblocks possessed atatic PHB segments and had good solubility but lacked sufficient crystallinity as solids. However, there are issues with atatic PHB which can have severe implications for biological properties such as biodegradability and biocompatibility (Kumagai et al. 1993; Shuai et al. 2000). Chen used such triblocks to form biodegradable nanoparticles for drug release, which showed better release characteristics than triblocks of PHB with poly(ethylene oxide), (PEO-PHB-PEO), (Chen et al. 2006). Low molecular weight PEGylated PHB triblocks also demonstrated self-assembly and formed strong hydrogels (Kerh et al. 2010).

In contrast to studies using synthetic PHB, Marchessault and coworkers used microbial PHB which are $100 \%$ isotatic, highly crystalline and insoluble in water. Diblock copolymers of this bacterial PHB with monomethoxy-PEG (mPEG) were synthesised using the dehydrating agent dicyclocarbodi-imide (DCC) and (dimethyleamino)pyridine (DMAP) as a catalyst (Figure 3). However, yields and reaction times were low (Marchessault and $\mathrm{Yu}$, 1999). Using the same PHB and mPEG-2000 components, but with bis(2-ethylehexanote) tin as a catalyst in a transesterification reaction resulted in yields of up to $77 \%$ within 60 minutes. This single-step synthesis of mPEGylated PHB proceeded with a concomitant depolymerisation to lower molecular weights, such that molecular weights of the resulting hybrids were reduced to approximately 2,300 to 7,300 (Ravanelle and Marchassault, 2002).

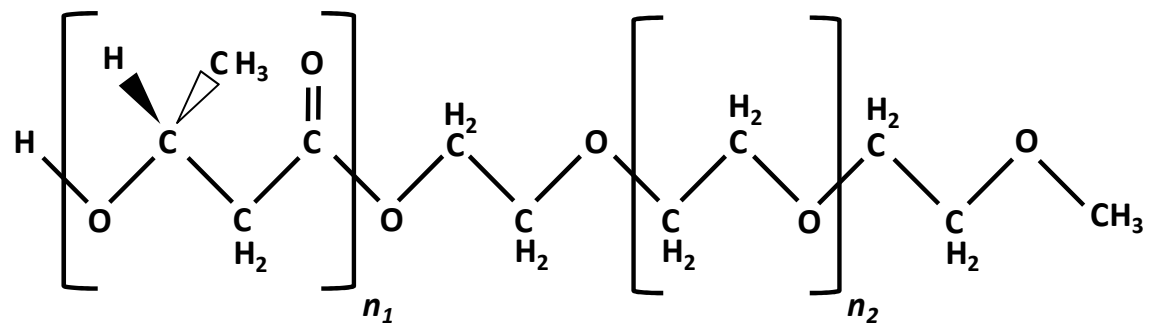

Fig. 3. Chemical formula for diblock copolymer of PHB $\left(n_{1}\right)$ and $\operatorname{MPEG}\left(n_{2}\right)$.

As transesterification is a random chain scission process, its application for PEGylation of PHAs can result in hybrids with wide molecular polydispersity. By manipulating the experimental conditions, mPEG in the final diblock ranged from 23 to $53 \%$ of the weight and these segments reduced the melting point of the PHB as well as crystallisation behaviour (Ravanelle and Marchassault, 2002). The synthesis of such low molecular weight PEGylated PHB supports their application as soluble therapeutics and for drug delivery.

\section{BioPEGylation of PHAs}

\subsection{Modulation of PHA composition using PEG}

While copolymerisation of Poly(3-hydroxybutyrate) with Poly(4-hydroxybutyrate), P(3HBco-4HB), results in copolymer with more favourable physiochemical and material properties than its $\mathrm{P}(3 \mathrm{HB})$ counterpart, yields are low. PEG is known to associate with phospholipids' head groups in cell membranes, and this promotes an increase in membrane fluidity and consequently ions and small metabolites permeation (Yamazaki \& Ito, 1990; Ingram \& Buttke, 1984). In 1996, Gross and coworkers working on the theory that adding PEG-200 to the cultivation medium of the microorganism Ralstonia eutropha during the production of $\mathrm{P}(3 \mathrm{HB}-\mathrm{co}-4 \mathrm{HB})$ would promote the synthesis of PHAs with an enhanced composition of 4- 
HBA units (Shi et al. 1996a). By extension of this reasoning, PEG modulated bioprocessing could also increase the diversity of monomeric units incorporated, potentially leading to the synthesis of new PHAs.

Shi et al. cultivated $R$. eutropha for the production of $\mathrm{P}(3 \mathrm{HB}-\mathrm{co}-4 \mathrm{HB})$ in Erlenmeyer flasks with PEG-200 loadings of 1 to $4 \%(\mathrm{w} / \mathrm{v})$. When cultivated on 4-hydroxybutyrate as carbon source, R. eutropha synthesised a random copolymer with 3-HBA and 4-HBA monomeric units. However, addition of only 2\% (w/v) PEG-200 to the bioprocessing significantly reduced the proportion of 3-HBA units from 34 to $11 \mathrm{~mol} \%$, with an increase in 4-HBA units from 66 to $86 \mathrm{~mol} \%$. Furthermore, this scl-PHA also contained approximately $3 \mathrm{~mol} \% 3-$ hydroxyvalerate units, $\mathrm{P}(3 \mathrm{HB}-\mathrm{co}-3 \mathrm{HV}-\mathrm{co}-4 \mathrm{HB})$, (Shi et al. 1996a). Steinbüchel and coworkers have compared the influence of PEG-200 to a variety of chemical inhibitors of fatty acid oxidation and the citric acid cycle to probe the influence of such polyethers on lipid and PHA synthesis in various Rhodococcus and Nocardia species (Alvarez et al. 1997). In this study they report that 0.2 to $5 \%$ (w/v) PEG-200 in the cultivation media caused decreases in scl-PHA contents in $R$. ruber, but under the conditions studied also stimulated greater incorporation of $3 \mathrm{HV}$ monomer units.

Foster and coworkers report that PHO produced by Pseudomonas oleovorans in the presence of 2\% (w/v) PEG-106 had a significant change in the relative proportions of its C6, C8 and C10 monomeric hydroxyalkanoate components (Sanguanchaipaiwong et al. 2004; Foster et al. 2005). Bioprocessing using another strain of P. oleovorans in the presence of PEG-200 and PEG-400 for the production of a $f c l$-PHA with some degree of unsaturated bonds in the side chain, also saw a change in its composition, with the C8 component increasing from 47 to 56 mol \% (Ashby et al. 2002). Results suggest that the presence of relatively low molecular weight PEGs in microbial growth media provided some measure of control over the PHA monomeric composition by promoting the main monomer in the carbon feed source. Therefore, $R$. eutropha cultivated with 4-hydroxybutyrate as carbon source incorporated more of these units in its scl-PHA, while different strains of $P$. oleovorans followed this trend for their $\mathrm{mcl}$ - and $f \mathrm{cl}$-PHAs when cultivated with octanoic and oleic acids respectively (Ashby et al. 2002; Foster et al. 2005).

\subsection{Modulation of PHA molecular weight using PEG}

In addition to influencing PHA composition, the presence of PEGs in the bioprocessing systems also affected the molecular mass of the biopolymers (Figure 4). With the exception of Azotobacter vinelandii UWD, PEGs with molecular weights greater than approximately 2,000 had no apparent impact on the molecular weights of the scl-PHAs synthesised by a variety of microbial species. However, PEGs with molecular weights below 2,000 supported PHA chain termination and resulted in PHA molecular weights significantly below those for scl-PHAs synthesised in the absence of PEGs. The lower the PEG molecular weight the greater the PHA molecular weight reduction (Figure 4).

PHB synthesis by R. eutropha with PEG-350 and its methoxy end-capped derivatives in the cultivation media have shown that capping of one hydroxyl group on the PEG chain had no apparent affect on the ability of PEG-350 to interact with polymer biosynthesis and effect molecular weight change. However, when both hydroxyl groups on the PEG were capped molecular weight reduction did not occur (Shi et al. 1996b). These results suggest that PEGs interact with the biosynthetic system, presumably through the PHA synthase, to increase the rate of sclPHA chain termination relative to propagation. 


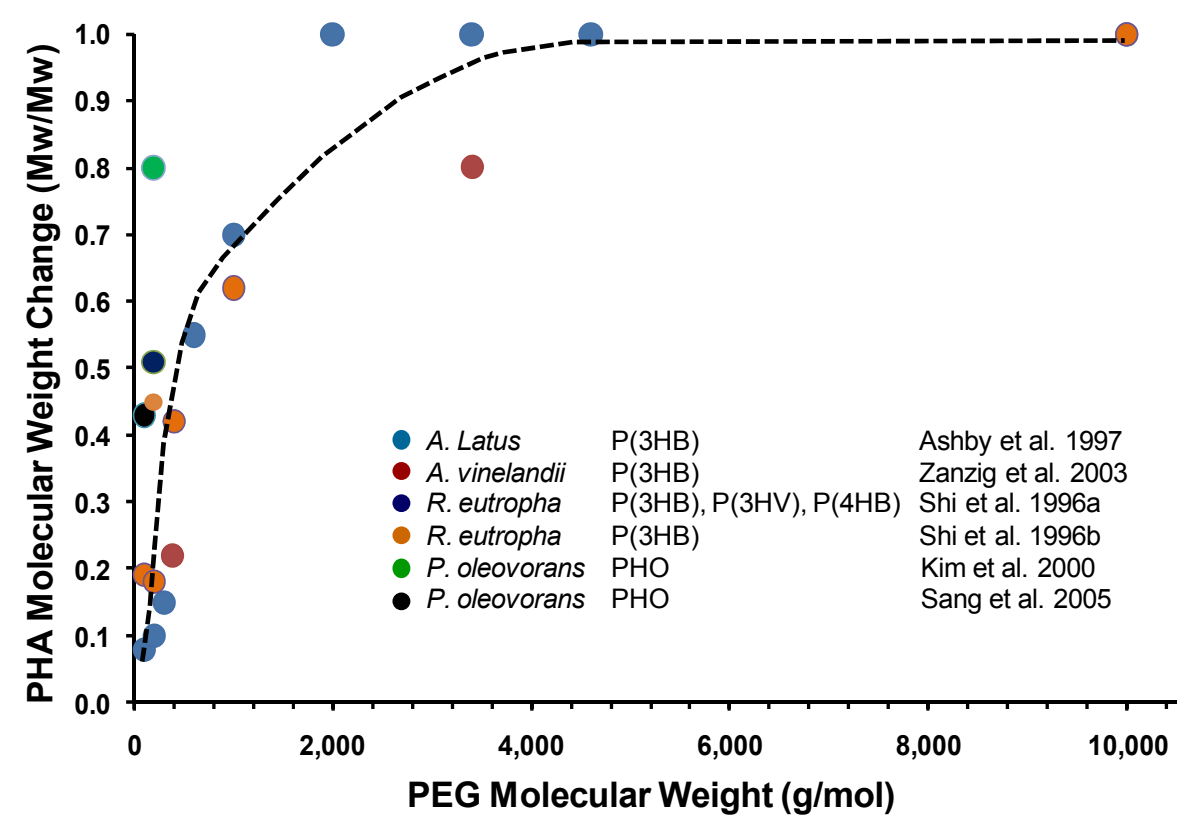

Fig. 4. Graph illustrating the relative change in molecular weight of PHAs due to presence of PEGs in the cultivation media.

Molecular weight is an important factor influencing the biodegradability and physicomechanical properties of a biomaterial. Consequently, it has been suggested that PEGs may also offer some degree of PHA molecular weight control, a so-called 'tunable switch' (Ashby et al. 1999). The addition of $2 \%$ (w/v) PEG-200 to cultivations of A. latus and R. eutropha at different growth stages showed that the PHB synthesised had bimodal distributions of comparatively high and low molecular weights. In contrast, additions of PEG-200 at the beginning of the cultivations produced PHB with low to high ratios of 1:0.10 and 1:0.19 for A. latus and R. eutropha respectively; these ratios increased to 1:7.33 and 1:3.17 when the PEG was added during the established growth phases (Ashby et al. 1999).

The addition of comparatively low molecular weight PEGs to bioprocessing systems using P. oleovorans and P. putida induced manipulation of mcl-PHAs molecular weight. Additions of $2 \%(\mathrm{w} / \mathrm{v})$ of PEG-106, PEG-200 or PEG-400 reduced the molecular weights of PHO synthesised by these species (Kim, 2000; Sanguanchaipaiwong et al. 2004). However, the impact appeared to be greater for $P$. oleovorans that $P$. putida with this $m c l$-PHA reduced to 40 and $80 \%$ respectively (Kim 2000).

\subsection{Biosynthesis of PHA-PEG natural-synthetic hybrid copolymers}

Modification of PHA molecular weight and composition during their biosynthesis is dependent upon the microbial species, the conditions of its cultivation and the molecular weight of PEG utilised, with PEGs possessing a relatively low degree of polymerisation influenced PHA compositions and reduced their molecular weights. Changes in molecular weight suggest that PEG molecules promote PHA chain scission. ${ }^{1} \mathrm{H}$ - and ${ }^{13} \mathrm{C}-\mathrm{NMR}$ analysis of PHAs from PEG modulated cultivations support this, with results proving the 
termination of PHA chains by the PEG chains (Madden et al. 1994; Shi et al. 2000b; Sangaunchaipaiwong et al. 2004; Foster et al. 2005). Thus, PHA chains are 'end-capped' with PEGs forming a new class of natural-synthetic hybrid block copolymers. This biological endcapping is referred to as 'bioPEGylation' (Foster, 2007).

Foster and coworkers have quantitatively monitored the concentrations of PEG-106 in cultivation media during the synthesis of PHO by $P$. oleovorans and for PHB production in $A$. latus. PEG-106 was also shown to be readily associated with the cell membranes and penetrated into the cytoplasm (Sanguanchaipaiwong et al. 2004; Foster et al. 2005; Sanguanchaipaiwong, 2007). ${ }^{13}$ C-labelled ethylene glycol (EG) was utilised by Shah et al. to probe the influence of these polyethers on the PHB synthetic pathway of R. eutropha. The results clearly showed that the EG units acted as PHB chain terminators (Shah et al. 2000a, 2000b).

\begin{tabular}{|c|c|c|c|c|c|c|c|c|c|c|c|c|c|}
\hline \multirow[b]{2}{*}{ Species } & \multirow{2}{*}{$\begin{array}{l}\text { EG } \\
62\end{array}$} & \multirow{2}{*}{$\begin{array}{l}\text { DEG } \\
106\end{array}$} & \multirow[b]{2}{*}{200} & \multirow[b]{2}{*}{300} & \multicolumn{6}{|c|}{ Polyethylene Glycol (PEG) } & \multirow[b]{2}{*}{4,600} & \multicolumn{2}{|r|}{ reference } \\
\hline & & & & & 350 & 400 & 600 & 1,000 & 2,000 & 3,400 & & 10,000 & \\
\hline \multicolumn{14}{|l|}{ sclPHA } \\
\hline Comomonas testosteroni ${ }^{* *}$ & YY & & & & & & & & & & & & Shah et al. 2000 \\
\hline Ralstonia eutropha * & $Y Y$ & & & & & & & & & & & & Madden et al. 1999; Shah et al. 2000 \\
\hline " $\quad *$ ** & & & Y Y & & & & & & & & & & Shi et al. $1996 \mathrm{a}$ \\
\hline " $\quad$ " * & & Y N & Y N & & Y N & Y N & & Y N & & & & N N & " " 1996b \\
\hline " $\quad$ * & & & Y N & & & & & & & & & & Ashby et al.1999 \\
\hline Alcaligenes latus * & & & Y N & & & & & & & & & & " " " " \\
\hline " " * & & $Y Y$ & $Y Y$ & $Y Y$ & & & $Y Y$ & $Y Y$ & Y N & & N N & N N & Ashby et al. 1997 \\
\hline " $\quad$ " * & & $Y Y$ & & & & Y N & & & N - & N- & $\mathrm{N}-$ & & Zanzig et al. 2003 \\
\hline Azotobacter vinelandii UWD & & $Y Y$ & & & & YN & & & N - & N- & $\mathrm{N}-$ & & " " " " \\
\hline $\begin{array}{l}\text { Pseudomonas oleovorans * } \\
\text { B14682 }\end{array}$ & & & YY & & & & YY & & & & & & Ashby et al. 2002 \\
\hline$s c / P H A+f c l$ PHA & & & YY & & & YY & & & & & & & \\
\hline P. oleovorans $B 778$ * & & & & & & & & & & & & & Ashby et al. 2002 \\
\hline$m c /$ PHA & & & YN & & & Y N & & & & & & & \\
\hline P. putida KT2442 ${ }^{\circ}$ & & & Y N & & & Y N & & & & & & & Kim 2000 \\
\hline P. oleovorans ATCC $29347^{\circ}$ & & $Y Y$ & YY & & & Y N & & & & & & & " " \\
\hline “ & & & & & & & & & & & & & Sanguanchaipaiwong et al. 2005, 2007 \\
\hline fc/PHA & & & Y N & & & Y N & & & & & & & \\
\hline$B 14683 \neq$ & & & & & & & & & & & & & Ashby et al. 2002 \\
\hline
\end{tabular}

Table 1. Summary of PHA bioPEGylation and/or molecular mass reduction due to the addition of various molecular weight PEGs to microbial cultivations for the production of PHAs. $(\mathrm{Y}=$ molecular mass reduction, $\mathrm{Y}=$ bioPEGylation, $\mathrm{N}=$ No effect, - not measured), *(3HB); ** (3HB)(4HB)(3HV); $190 \mathrm{~mol} \%(3 \mathrm{HB})+10 \mathrm{~mol} \%$ fclPHA; ${ }^{\circ} \mathrm{fclPHA}, \neq \mathrm{PHO}$

The influence of PEG molecular weights on PHA hybrid biosynthesis and molecular weight reduction for a number of species is summarised in Table 1. From Table 1 it can be clearly seen that bioPEGylation of PHA chains does not always occur and appears to be favoured in cultivation systems where the PEG molecular weight is 300 or below. However, this is not entirely surprising given the original premise that these polyethers would support small molecule penetration into the cell.

From the summary in Table 1, we can speculate that PEGs with molecular weights up to 300 facilitate PHA chain scission as well as the synthesis of bioPEGylated hybrids. PEG molecular weights between 300 and 2,000 may support fluidity of the cell membrane but due to their large size, they fail to enter the cell and do not act as chain terminating agents. Finally, PEGs with molecular masses exceeding 2,000 do not appear to influence PHA 
properties. While PEGs with molecular weights between 300 and 2,000 supported PHA composition and molecular mass control, these influences may be a function of the additives influence on cell viability.

\subsection{Influence of PEGs on PHA bioprocessing parameters}

Cell yields for R. eutropha for PHB production decreased by $30 \%$ as the concentration of PEG-200 in growth media increased from 1 to $4 \%$ (w/v). In addition, the sclPHA yield also decreased by $50 \%$. In companion experiments, the viability of this species was also shown to be reduced with the addition of 1 to 10\% (w/v) of PEG-200 or PEG-400 (Shi et al. 1996b). Thus, higher concentrations of these comparatively low molecular weight PEGs in the microbial growth medium, the greater the reduction in cell viability. In contrast, increases in PEG molecular weight had comparatively less influence on cell viability. Thus, the addition of similar loadings of PEG-10,000 had no apparent effect on $R$. eutropha cell viability (Shi et al. 1996b). Studies reporting reductions in cell viability during PEG modulated cultivations for PHA production support the theory proposed here that PEGs of molecular weights between 300 and 2,000 influences PHA molecular mass and composition through modifications to cell viability in the bioprocessing system. In contrast, PEGs with molecular weights below approximately 300 modify PHA synthesis by acting as chain terminating agents forming natural-synthetic hybrids.

With the exception of PEG-10,000, the presence of PEGs in the cultivation media reduced the yield of scl-PHAs in R. eutropha. This was partly a consequence of their influence on cell mass (Shi et al. 1996b). Similar studies using A. latus have shown that PEGs with molecular weights between 106 and 600 had a greater impact on polymer synthesis compared to their higher molecular weight counterparts $(1,000$ - 10,000), with PHB productivity also being reduced depending on PEG concentration in the cultivation medium (Ashby et al. 1997). Thus, while 5\% (w/v) PEG-106 in the growth media resulted in no PHA synthesis, 10\% $(\mathrm{w} / \mathrm{v})$ of PEG-10,000 still showed some biopolymer synthesis, although the impact was greater than that observed when the studies were conducted with R. eutropha (Ashby et al. 1997). Similarly, additions to cultivations of A. vinelandii UWD of $2 \%(\mathrm{w} / \mathrm{v})$ of various PEGs from PEG-106 to PEG-3400 also resulted in variable polymer yields (Zanzig et al. 2003). P(HB-co-HV) synthesis by $R$. ruber was influenced by the presence of $0.2-5 \%(\mathrm{w} / \mathrm{v})$ PEG-200, with a reduction in scl-PHA yield of up to 50\% (Alvarez et al. 1997).

Such variations in polymer yields are obviously due to the influence of PEGs on the bioprocessing systems. However, the utilisation of Erlenmeyer flasks in these studies limits their value. Foster and coworkers have cultivated A. latus for the production of PHB in the presence of $2 \%(\mathrm{w} / \mathrm{v})$ PEG-106 using Braun $5 \mathrm{~L}$ bioreactors, which provides greater control over cultivation parameters. In these studies PEG was demonstrated to support an increase in cell mass $(12 \%)$ as a consequence of the additional carbon, but through reduced microbial viability a reduction in cellular PHA synthesis was measured (9\%), (Sanguanchaipaiwong, 2007). This increase in cell mass is consistent with the studies of Shah et al. who reported that EG was metabolised by R. eutropha for the production of PHB (Shah et al. 2000a)

Microbial PHA synthesis is species dependent; the diversity of $f c l$ - and mcl-PHAs are significantly greater than their sclPHA companions. Kim investigated the influence of PEG200 on the synthesis of mcl-PHAs by P. oleovorans and P. putida and reported that PHO yield was reduced with increasing PEG concentration, while at 8\% PEG-200 loading no PHO synthesis was found (Kim, 2000). Using the same strain of P. oleovorans cultivated in the 
presence of PEG-106, Foster and coworkers reported an increase in cell concentration but a decrease in PHO yield (Sanguanchaipaiwong et al. 2004). Similarly, the addition of PEG-200 or PEG-400 to another strain of P. oleovorans (B-778) primarily producing PHB but mixed with $10 \mathrm{~mol} \%$ of a $f c l$ PHA, also showed an increase in cell mass but no change in polymer yield. In contrast, other strains of P. oleovorans producing PHB (B-14682) and a $f c l$ PHA (B14683), showed no apparent change in cell mass but did show a reduction in their polymer yields (Ashby et al. 2002). Thus, the influence of PEGs on PHA synthesis also has a degree of species dependence.

\section{Properties and potential}

\subsection{Physiochemical and material properties}

The majority of current studies in this emerging field have investigated the influence of PEGs on PHA synthesis, with a focus on their potential to control composition and molecular mass of these biopolyesters. Thus, PEG modulated bioprocessing of PHAs may offer a technique to control PHA physiochemical and material properties. In addition, characterisations of natural-synthetic hybrids of bioPEGylated PHAs suggest that these hybrids may also possess additional properties when compared to their PHA counterparts (Zanzig et al. 2003; Foster et al. 2005). Concurrent cultivations of A. latus from the same inoculums have shown that the PHB-co-PEG106 hybrid, while possessing a lower molecular weight compared to its PHB counterpart, also had significantly different physiochemical and material properties. In particular the elongation to break increased from 8.4 to $20.6 \%$ (Sanguanchaipaiwong, 2007). In contrast, the change in elongation-to-break for bioPEGylated mcl-PHA was not as noticeable, $580( \pm 9.4)$ and $540( \pm 8.3) \mathrm{MPa}$ for PHO and PHO-co-PEG106 respectively.
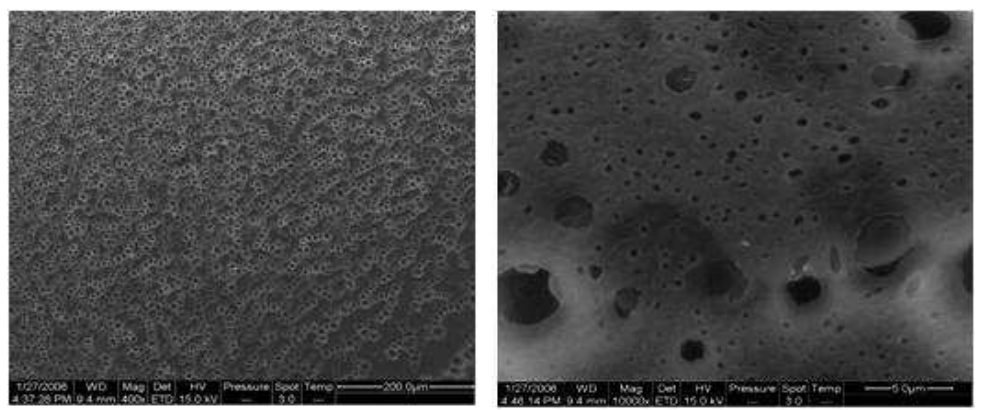

Fig. 5. SEMs of PHO-co-PEG106 films fabricated though solvent casting in the presence of humidity reveal the presence of pores not observed with PHO.

Small angle neutron scattering (SANS) was utilised to probe the chain conformations of protonated and deuterated mcl-PHA samples as well as their bioPEGylated hybrids. The comparatively small hydrophilic PEG group terminating much longer hydrophobic PHA chains exhibited no apparent influence on chain conformation when solvated in chloroform (Foster et al. 2008). However, with the addition of microquantities of water to the bioPEGylated PHA solutions, the hybrids formed stable microcrystalline suspensions. Consequently, when processed under humid airflow, bioPEGylated PHAs exhibited a degree of self-assembly resulting in the formation of disordered microporous films (Figure 
5), (Foster et al. 2005; Sanguanchaipaiwong, 2007). Microporous and honeycomb films are of considerable interest based on their biotechnological and biomedical potential, such as immobilisation of biomolecules (Stenzel, 2002; Nishikawa et al. 1999).

\subsection{Biological properties}

Scholz and coworkers investigated the interactions of various bioPEGylated PHB samples with skin melanoma and human breast adenocarcinoma cells (Zanzig et al. 2003). In this study, cell adhesion was significantly affected by the presence of the PEG hydrophilic end groups, with reductions in cell attachments to hybrids ranging from 22 to $74 \%$. The greatest reduction was found with PHB-co-PEG106, (Zanzig et al. 2003).

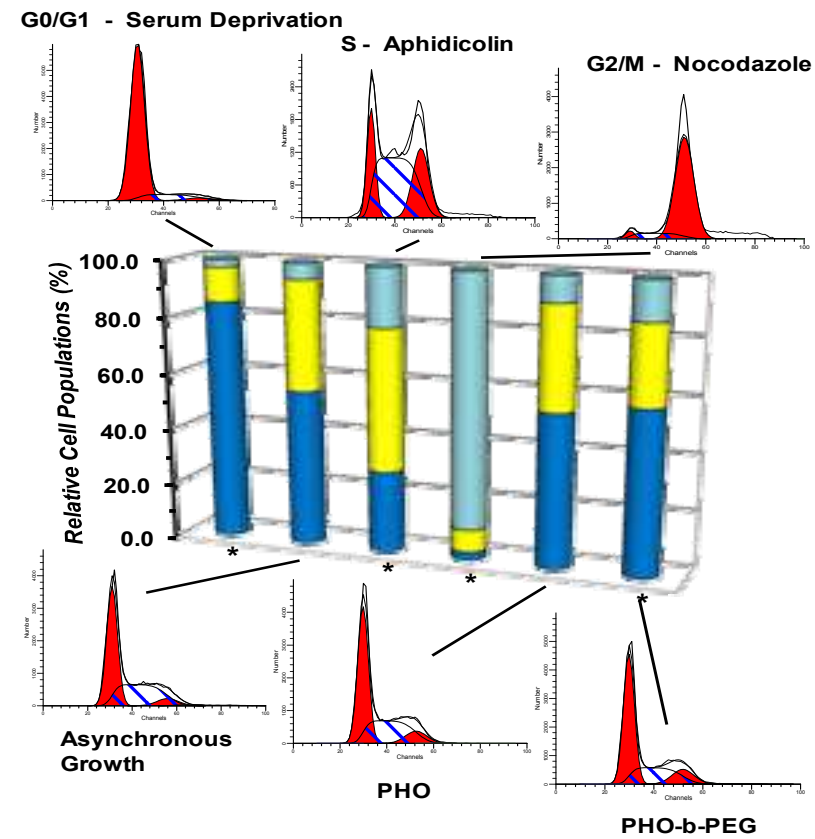

Fig. 6. Summary of relative cell growth populations determined from FACS sorting histograms, expressed as percentages of total populations. BioPEGylated PHO films showed statistically significant differences for cell populations in the G2/M (p<0.1) and $S$ (p < 0.001) phases, when compared to the same population cultivated in the absence of biomaterials. ( ${ }^{*}$ indicates statistically significant differences from asynchronous growth).

Figure 6 shows the changes in cell cycle when myoblastic satellite stem cells were cultivated in the presence of PHO and its bioPEGylated hybrid. The hybrid showed comparatively small but significant changes in the DNA population distributions for the stem cells compared to PHO, with significant differences between the two PHAs. Hence, the cycle of stem cell differentiation from G0-G1 through S to G2-M phase proceeded in the presence of $\mathrm{PHO}$ and its hybrid, but with significant differences in the respective populations of cells at these phases when compared to asynchronous growth, with the PHO-co-PEG106 showing the greatest deviation (Figure 6), (Marcal et al. 2008). 


\section{Conclusions and future trends}

Conjugations of the scl-PHA, PHB with various PEGs, i.e, PEGylation, have been performed through chemical routes. The resulting diblocks and triblocks of this PHA have a low molecular weight and possess potential as pharmaceutical agents and polymers for injectable hydrogels or nanoparticles for drug delivery.

Strategic additions of PEGs to microbial cultivations for the production of $s c l$ - and $m c l$-PHAs not only permit a degree of control in PHA molecular mass and composition but may also provide routes for BioPEGylation. While bioPEGylated PHAs have a significantly reduced molecular weight compared to their PHA counterparts produced in the absence of PEG, they are considerable greater than the PEGylated counterparts and retain their material properties. Nevertheless, bioPEGylation permits significant changes in physiochemical and material properties, with subsequent implications for their material processing. Furthermore, the presence of hydrophilic end groups has significant implications for the biological properties of these hybrids, suggesting they may serve as novel biomaterials in their own right.

In general, PEGs with molecular weights below approximately 300-600 apparently increase membrane fluidity, supporting bioPEGylation. While PEGs with molecular masses from 600 to 2,000 facilitate PHA molecular mass and composition control, without bioPEGylation; PEGs above 2,000 appear to have little or no effect. For those PEGs exerting some degree of control over PHA synthesis, polymer yields are significantly reduced. Furthermore, this review suggests that, as with PHA biosynthesis, the influence of PEGs in cultivations systems may be strain specific.

Despite the molecular weight disparities between the hydrophobic PHA chain and it's comparatively tiny hydrophilic PEG terminal in bioPEGylated PHAs, there is sufficient evidence to suggest that the hybrid has subtly different physiochemical and material properties. Thus, work in this field is consistent with current trends to increase the diversification of PHAs and their conjugates (Hazer and Steinbüchel, 2007). Future work requires more detailed study of PHA-co-PEG hybrids as biomaterials, as well as investigations in the potential application of PEGs to bioprocessing technology for the production control of PHAs.

While PEGylation and bioPEGylation studies are still in their infancy, results to date suggest that these natural-synthetic hybrids have great potential as medical biomaterials.

\section{References}

Alvarez HM, Kalscheuer R, Steinbüchel A (1997) Accumulation of storage lipids in species of Rhodococcus and Nocardia and effect of inhibitors and polyethylene glycol. Fett Lipid 99:239-246.

Ashby RD, Shi F, Gross RA (1997) Use of poly(ethylene glycol) to control the end group structure and molecular weight of poly(3-hydroxybutyrate) formed by Alcaligenes latus DSM 1122. Tetrahedron 53:15209-15223.

Ashby RD, Shi F, Gross RA (1999) A tunable switch to regulate the synthesis of low and high molecular weight microbial polyesters. Biotech Bioeng 62:106-113.

Ashby RD, Solaiman DKY, Foglia TA (2002) Poly(ethylene-gycol)-mediated molar mass control of short-chain- and medium-chain-length poly(hydroxyalkanoates) from Pseudomonas oleovorans. Appl Microbiol Biotechnol 60:154-159. 
Anderson AJ, Dawes EA (1990) Occurance, metabolism, metabolic role, and industrial uses of bacterial polyhydroxyalkanoates. Microbiol Rev 54:450-472.

Bondy PK, Blom WL, Whitner VS, Farrar BW (1949) Studies of the role of the liver in human carbohydrate metabolism by venous catheter technic. II. Patients with diabetic ketosis, before and after the administration of insulin. J Clin Invest 28:1126-1125.

Borgens RB, Bohnert D. (2001) Rapid recovery from spinal cord injury after subcutaneously administered polyethylene glycol. J Neurosci Res 66(6):1179-1186.

Chen C, Yu CH, Cheng YC, Yu PH, Cheung MK. (2006) Biodegradable nanoparticles of amphiphilic triblock copolymers based on poly(3-hydroxybutyrate) and poly(ethylene glycol) as drug carriers. Biomaterials 27:4804-4814.

Chen G.-Q, Wu Q. (2005) The application of polyhydroxyalkanoates as tissue engineering materials. Biomaterials 26:6565-6578.

Cheng GX, Cai ZJ, Wang L. (2003) Biocompatibility and biodegradability of poly(hydroxybutyrate)/poly(ethylene glycol) films, J Mater Sci-Mater M 14,1073-8.

Doi Y, Microbial Polyesters. New York: VCH Publishers; 1990

Foster LJR, Tighe BJ (1995) Enzymatic assay of hydroxybutyric acid monomer formation in poly( $\beta$-hydroxybutyrate)degradation studies. Biomaterials 10:314-343.

Foster LJR, Fuller RC, Lenz RW (1996) Activities of extracellular and intracellular depolymerases of polyhydroxyalkanoates. In: Ottenbrite RM, Huang SJ, Park K. Hydrogels and biodegradable polymers for bioapplications. ACS books, Washington DC, pp 68-92.

Foster LJR (2000) The structural organisation of polyhydroxyalkanoate inclusion bodies. In: Scholz C, Gross RA. Polymers from renewable resources: biopolyesters and biocatalysis. ACS books, Washington DC, pp 42-66.

Foster LJR, Sanguanchaipaiwong V, Gabelish CL, Hook J, Stenzel M (2005) A naturalsynthetic hybrid of polyhydroxyoctanoate-diethylene glycol: biosynthesis and properties. Polymer 46:6587-6594.

Foster LJR, Schwahn D, Pipich, V, Holden, PJ, Richter, D. (2008) SANS characterisation of polyhydroxyalkanoates and their bioPEGylated hybrids in solution. Biomacromolecules 9:314-320.

Hängii UJ, (1995) Requirements of bacterial polyesters as future substitutes for conventional plastics for consumer goods. FEMS Microbiol Rev 16:213-220.

Harris (1992) Introduction to biotechnical and biomedical applications of poly(ethylene glycol). In: Harris JM, (ed) Poly(ethylene glycol) chemistry: biotechnical and biomedical applications. Plenum press, new York, pp 1-14.

Harris JM, Chess RB (2003) Effect of PEGylation on Pharmaceuticals. Nat Rev Drug Discuss 3:214-221.

Hazer B, Steinbüchel A (2007) Increased diversification of polyhydroxyalkanoates by modification reactions for industrial and medical applications. Appl. Microbiol Biotechnol 74:1-12.

Ingram LO, Buttke TM (1984) Effects of alcohols on microorganisms. Adv Micro Phys 25:253300.

Kerh LL, Jing-ling Z, Jun L. (2010) Elucidating rheological property enhancements in supramolecular hydrogels of short poly[(R,S)-3-hydroxybutyrate]-based amphiphilic triblock copolymer and a-cyclodextrin for injectable hydrogel applications. Soft Matter 6:2300-2311. 
Krause TL, Bittner GD. (1990) Rapid morphological fusion of severed myelinated axons by polyethylene glycol. PNAS 87(4):1471-1475.

Kim DY, Kim YB, Rhee YH (2000) Evaluation of various carbon substrates for the biosynthesis of polyhydroxyalkanoates bearing functional groups by Pseudomonas putida. Int J Bio Macromol 28:23-29.

Kim O (2000) Biological effects of poly(ethylene glycol) on microbial poly( $\beta$ hydroxyalkanoates) produced by pseudomonads microorganisms. J Polym Res 7:9196.

Kim YB, Lenz, RW, Fuller RC (1995) Poly-3-hydroxyalkanoates containing unsaturated repeating units produced by Pseudomonas oleovorans. Macromolecules 25:1852-1857.

Kumagai Y, Doi Y (1993) synthesis of a block copolymer of poly(3-hydroxybutyrate) and poly(ethyelene glycol) and its application to biodegradable polymer blends. J Polym Environ 1:81-87.

Laverty PH, Leskovar A, Breur GJ, Coates JR, Bergman RL, Widmer WR, Toombs JP, Shapiro S, Borgens RB. (2004) A preliminary study of intravenous surfactants in paraplegic dogs: Polymer therapy in canine clinical SCI. J Neurotrauma 21, 12, 17671777.

Lenz RW (1993) Biodegradable polymers. Adv Polym Sci 107:1-40.

Lenz RW, Marchessault RH (2005) Bacterial polyesters: biosynthesis, biodegradable plastics and biotechnology. Biomacromolecules 6:1-8.

Madden LA, Anderson AJ, Shah DT, Asrar J (1999) Chain termination in polyhydroxyalkanoate synthesis: involvement of exogenous hydroxyl-compounds as chain transfer agents. Int J Biol Macromol 25:43-53.

Marçal H, Wanandy NS, Sanguanchaipaiwong V, Woolnough, CE, Lauto A, Mahler SM, Foster LJR. (2008) BioPEGylation of polyhydroxyalkanoates: influence on properties and satellite stem cell cycle. Biomacromolecules 9(10):2719-2726.

Marchessault RH, Yu G.-E. (1999) Polym. Prepr. 40:527-528.

Martin DP, Peoples OP, Williams SF, Zhong LH. (1999) Nutritional and therapeutic uses of 3-hydroxyalkanoate oligomers, US Patent Appl 359086.

Nakamura S, Doi Y, Scandola M (1992) Microbial synthesis and characterisation of poly(3hydroxybutyrtae-co-4-hydroxybutyrate). Macromolecules 25:4237-4241.

Nishikawa T, Nishida J, Ookura R, Mishimura S-I, Wada S, Karino T, Shimomura M (1999) Honeycomb-patterned thin films of amphiphillic polymers as cell culture substrates. Mater Sci Eng C8:495-500.

Ravanelle F, Marchessault RH (2002) One-step synthesis of amphiphillic diblock copolymers from bacterial poly([R]-3-hydroxybutyric acid). Biomacromolecules 3:1057-1064.

Reusch RN, Sparrow AW, Gardiner J (1992) Transport of poly-beta-hydroxybutyrate in human plasma. Biochim Biophys Acta 1123:33-40.

Sanguanchaipaiwong V, Gabelish CL, Hook J, Scholz C, Foster LJR (2004) Biosynthesis of natural-synthetic hybrid copolymers: polyhydroxyoctanoate-diethylene glycol. Biomacromolecules 5:643-649.

Sanguanchaipaiwong V (2007) Biosynthesis and characterisation of polyhydroxyalkanoate based natural-synthetic hybrid copolymers. Ph.D. thesis, University of New South Wales, Sydney, Australia. 
Shah DT, Tran M, Berger PA, Aggarwal P, Asrar J, Madden LA, Anderson AJ (2000a) Synthesis and Properties of hydroxyl-terminated poly(hydroxyalkanoates). Macromolecules 33:2875-2880.

Shah DT, Berger PA, Tran M, Asrar J, Madden LA, Anderson AJ (2000b) Synthesis of poly(3hydroxybutyrate) by Ralstonia eutropha in the presence of ${ }^{13} \mathrm{C}$-labelled ethylene glycol. Macromolecules 33:6624-6626.

Shi F, Ashby R, Gross RA (1996a) Use of poly(ethylene glycol) to regulate poly(3hydroxyybutyrate) molecular weight during Alcaligenes eutrophus cultivations. Macromolecules 29:7753-7758.

Shi F, Gross RA, Rutherford DR (1996b) Microbial polyester synthesis: effects of poly(ethylene glycol) on product composition, repeat unit sequence, and end group structure. Macromolecules 29:10-17.

Shuai XT, Jedlinski Z, Luo Q, Nozirov F (2000) Synthesis of novel block copolymers of poly(3-hydroxybutyric acid) with poly(ethyelene glycol) through anaionic polymerisation. Chin J Polym Sci. 18:19-23.

Steinbüchel A (1996) PHB and other polyhydroxyalkanoic acids. In: Rehm H-J, Reed G, Pühler A, Stadler P (ed) Biotechnology John Wiley \& Sons, Weinheim, vol 6. pp 403-464.

Steinbüchel A, Füchtenbusch B (1998) Bacterial and other biological systems for polyester production. TIBTECH 16:419-427.

Steinbüchel A (2001) Perspectives for biotechnological production and utilisation of biopolymers: metabolic engineering of polyhydroxyalkanoate biosynthesis pathways as a successful example. Macromol Biosci 1:1-24.

Steward A (2005) Polymers under one umbrella. Chem Ind 18/04/05 18-20.

Stenzel M (2002) Formation of regular honeycomb-patterned porous films by selforganisation. Aust J Chem 555239-243.

Wu, Q.; Wang Y. \& Chen, G-Q. (2009) Medical application of microbial biopolyesters polyhydroxyalkanoates. Artificial Cells Blood Sub Biotech 37, 1, 1-12.

Yamazaki M, Ito T (1990) Deformation and instability in membrane structure of phospholipid vesicles caused by osmophobic association - mechanical stress model for the mechanism of poly(ethylene glycol) induced membrane fusion. Biochemistry 29:1309-1314.

Yasin M, Tighe BJ (1993) Strategies for the design of biodegradable polymer systems manipulation of polyhydroxybutyrate based materials. Plast Rubb Compos Proc Appl 19:15-27.

Zanzig J, Marimuthu B, Werka J, Scholz C (2003) Investigation of the impact of poly(ethylene glycol)-modulation of poly( $\beta$-hydroxybutryate) syntheses on cell interactions of the resulting polymers. J Bioact Compat Polym 18:339-354. 


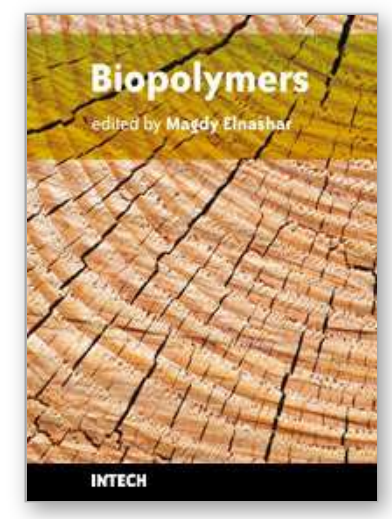

\author{
Biopolymers \\ Edited by Magdy Elnashar
}

ISBN 978-953-307-109-1

Hard cover, 612 pages

Publisher Sciyo

Published online 28, September, 2010

Published in print edition September, 2010

Biopolymers are polymers produced by living organisms. Cellulose, starch, chitin, proteins, peptides, DNA and RNA are all examples of biopolymers. This book comprehensively reviews and compiles information on biopolymers in 30 chapters. The book covers occurrence, synthesis, isolation and production, properties and applications, modification, and the relevant analysis methods to reveal the structures and properties of some biopolymers. This book will hopefully be of help to many scientists, physicians, pharmacists, engineers and other experts in a variety of disciplines, both academic and industrial. It may not only support research and development, but be suitable for teaching as well.

\title{
How to reference
}

In order to correctly reference this scholarly work, feel free to copy and paste the following:

Leslie John Ray Foster (2010). PEGylation and BioPEGylation of Polyhydroxyalkanoates: Synthesis, Characterisation and Applications, Biopolymers, Magdy Elnashar (Ed.), ISBN: 978-953-307-109-1, InTech, Available from: http://www.intechopen.com/books/biopolymers/pegylation-and-biopegylation-ofpolyhydroxyalkanoates-synthesis-characterisation-and-applications

\section{INTECH}

open science | open minds

\section{InTech Europe}

University Campus STeP Ri Slavka Krautzeka 83/A 51000 Rijeka, Croatia Phone: +385 (51) 770447

Fax: +385 (51) 686166 www.intechopen.com

\section{InTech China}

Unit 405, Office Block, Hotel Equatorial Shanghai No.65, Yan An Road (West), Shanghai, 200040, China 中国上海市延安西路65号上海国际贵都大饭店办公楼405单元 Phone: +86-21-62489820

Fax: $+86-21-62489821$ 
(C) 2010 The Author(s). Licensee IntechOpen. This chapter is distributed under the terms of the Creative Commons Attribution-NonCommercialShareAlike-3.0 License, which permits use, distribution and reproduction for non-commercial purposes, provided the original is properly cited and derivative works building on this content are distributed under the same license. 